\title{
LAS OPERACIONES DE PROTECCIÓN
}

Mayo E.M.C. José María Carranza ${ }^{1}$

"Mira por tus soldados como miras por un recién nacido; así estarán dispuestos a seguirte hasta los valles más profundos; cuida de tus soldados como cuidas de tus queridos hijos y morirán gustosamente contigo". (Sun Tzu)

\section{RESUMEN}

La planificación y ejecución de todas las operaciones militares siempre han contemplado entre sus aspectos más relevantes, el concepto de Seguridad. Sin embargo, la evolución de la doctrina, fruto del permanente cambio que caracteriza al arte de la guerra, ha vuelto más compleja la noción tradicional de la Seguridad y ha incorporado la idea de la Protección.

Este precepto va mucho más allá de la mera observancia y cumplimiento de las medidas de seguridad de las instalaciones, medios y tropas o de la ejecución de tareas tácticas destinadas a proporcionar la alerta temprana sino que también contempla todas las actividades necesarias para proteger y asegurar el poder de combate propio, el personal, los equipos e información de las acciones del enemigo.

Estos nuevos conceptos doctrinarios han tenido su origen en los primeros años del siglo XXI, cuando la creciente complejidad de la guerra y las diferentes capacidades tecnológicas al alcance de un enemigo no convencional, llevaron al ejército de los EE.UU. y luego a las fuerzas de la OTAN, a redefinir los conceptos de Seguridad y Protección.

Palabras claves: Seguridad, Protección, Funciones de combate, Planeamiento, Operaciones militares, Doctrina militar.

\begin{abstract}
The planning and execution of all military operations have always considered the concept of Security among its most relevant aspects. However, the evolution of the doctrine, the result of the permanent change that characterizes the art of war, has made the traditional notion of Security more complex and has incorporated the idea of Protection.

This precept goes far beyond mere observance and compliance with the security measures of the facilities, equipment and troops or the execution of tactical tasks aimed at providing early warning but also includes all the necessary activities to protect and ensure the own combat power, personnel, equipment and information of the enemy's actions.

These new doctrinal concepts have had their origin in the first years of the 21 st century, when the increasing complexity of the war and the different technological capabilities within reach of an unconventional enemy led the US Army and then to NATO forces, to redefine the concepts of Security and Protection.
\end{abstract}

Keywords: Security, Protection, War fighting functions, Planning, Military operations, Military doctrine.

\footnotetext{
1 josemacarranza@yahoo.com.ar EJÉRCITO DE ARGENTINA
} 


\section{Introducción}

Para arribar a una idea clara de que significa la Protección, es necesario mencionar primeramente que la doctrina militar vigente a nivel global ha contemplado ya desde la Segunda Guerra Mundial, el concepto de Seguridad, a la cual se puede definir como "el conjunto de actividades encaminadas a anular o reducir la eficacia de cualquier clase de acción adversaria hostil, sorpresiva, lo que incluye, especialmente, cualquier integrante del espectro de la amenaza aérea, blindada y mecanizada" (Ejército Ecuatoriano, 2015, pág. 24)

Por ello, esta definición se ha convertido en una de las premisas que todo comandante militar debe observar a la hora de planificar y ejecutar cualquier operación militar; consecuentemente, esta idea de Seguridad se ha convertido en uno de los denominados Principios de la Guerra o de la Conducción Militar, a los que doctrinariamente se define como "basamentos o causas de validez general para la conducción militar, que han sido aplicados por los grandes estrategas; deducidos y analizados por pensadores, estudiosos de la guerra y autores militares a través del tiempo, hasta constituir principios en razón a que la historia militar ha demostrado que su reiterada y correcta aplicación ha conducido a los ejércitos normalmente a la victoria" (Ejército Ecuatoriano, 2015, pág. 15) y en otros casos, en uno de los sistemas componentes de los llamados Sistemas Operativos del Campo de Batalla (S.O.C.B): mando y control, inteligencia, maniobra, seguridad, guerra electrónica, apoyo de fuego, apoyo de ingenieros y apoyo logístico. (Ejército Ecuatoriano, 2015, pág. 22)

Asimismo, la Seguridad se ha convertido en sí misma, en una operación complementaria cuya finalidad es la de brindar la alerta temprana, proporcionar tiempo y espacio para la reacción de la propia fuerza, preservar las propias instalaciones y tropas del accionar enemigo y negar información. (Ejército Argentino, 2015, pág. 191)

Sin embargo, el constante desarrollo tecnológico en materia de armamento, la aparición de la denominada "guerra de información", la ciberguerra y la posibilidad de acceder a múltiples capacidades de ataque por parte de organizaciones armadas no tradicionales, ha llevado a que el tradicional concepto de Seguridad no sea suficiente para lograr el adecuado grado de protección de las fuerzas y sus recursos críticos, los que ahora incluyen valores intangibles como la información y la moral de combatientes y civiles.

\section{¿Qué es Protección?}

Debido a la problemática que señalaba el tradicional concepto de Seguridad, que ahora se mostraba insuficiente, en la primera década del siglo XXI, el ejército de los EE.UU amplía la noción existente de Protección y desarrolla el concepto de las denominadas "Force Protection Operations" (Operaciones de Protección de la Fuerza), explicitado y desarrollado en el reglamento de "Protection" (FM 3-37) del año 2009: "La protección es la preservación de la efectividad del personal militar y no militar relacionado con la misión, equipos, instalaciones, información e infraestructura desplegados o ubicados dentro o fuera de los límites de un área operativa dada".

Esta nueva teoría es también desarrollada y aplicada en el ámbito de la OTAN, que la aprueba y pone en práctica a través del reglamento "Doctrina Conjunta Aliada para la Protección de la Fuerza" (AJP-3.14) del año 2015, el cual conceptualiza a la Protección como: "Son las medidas y medios para minimizar la vulnerabilidad del personal, las instalaciones, el equipo, el material, las operaciones y las actividades frente a las amenazas y los peligros a fin de preservar la libertad de acción y la eficacia operativa, contribuyendo así al éxito de la misión".

Ambas definiciones permiten visualizar que la protección va más allá de asegurar las propias fuerzas e instalaciones, sino que incluyen también la información, misión y operaciones, además, la idea tradicional de Seguridad y sus tareas inherentes, pasan a ser un componente fundamental de las operaciones de Protección.

Asimismo, la incorporación de la información como valor trascendental a proteger, implica que las medidas protectoras vayan más allá del campo de batalla tridimensional conocido por todos y que a partir de ahora, el ciberespacio, las redes sociales y los medios de comunicación se constituyan en nuevas zonas de combate. (Berkebile, 2016, pág. 141)

Del análisis de la doctrina estadounidense y de la OTAN, se puede visualizar que el concepto de Protección tiene dos grandes acepciones, ya que por un lado es considerada una de las funciones de combate, las que "proporcionan una forma eficaz para que los estados mayores identifiquen y relacionen las tareas que cada misión impone y determinen y seleccionen los sistemas y organizaciones más adecuadas para cumplirlas, de manera de asegurar que todos los aspectos relacionados con la conducción de las operaciones sean abordados" (Ejército Argentino, 2015, pág. 23) y por otro, es uno de los elementos del poder de combate (U.S. Army, 2009, pág. 9). 


\section{COMO ELEMENTO DEL} PODER DE COMBATE

- Preservación de la efectividad y supervivencia del personal, el equipo, las instalaciones, la información y la infraestructura (militar y no militar) relacionados con la misión y desplegados dentro o fuera de los límites del área operacional.

\section{COMO FUNCIÓN DE COMBATE}

- Conjunto de tareas y sistemas relacionados entre sí que preservan la Fuerza para que los comandantes puedan aplicar el máximo poder de combate en el cumplimiento de la misión.

Figura 1. Conceptos de Protección. (Fuente: elaboración propia).

Para superar la posible confusión que ambas definiciones pueden generar, se debe considerar a la Protección como elemento del poder de combate cuando se entienden y visualizan todas las actividades, acciones y efectos disponibles y necesarios para llevarla a cabo. Estas tareas o efectos pueden alcanzarse si se combinan con los otros cinco elementos del poder de combate: Movimiento y maniobra, Fuegos de apoyo, Sostenimiento, Inteligencia, y Comando y control. (U.S. Army, 2009, pág. 10)
Asimismo, si se considera la Protección como función de combate esta servirá para enfocarse en que tareas son relevantes e integrarlas y sincronizarlas dentro del proceso de las operaciones; además, describe claramente las doce tareas y sistemas que deben analizarse, controlar y evaluar durante la planificación y ejecución de las operaciones.

Además, bajo la guía de los principios que la conducen y combinando las diferentes formas de llevar a cabo la protección, se podrán desarrollar las tareas con mayor eficiencia y mejores resultados.
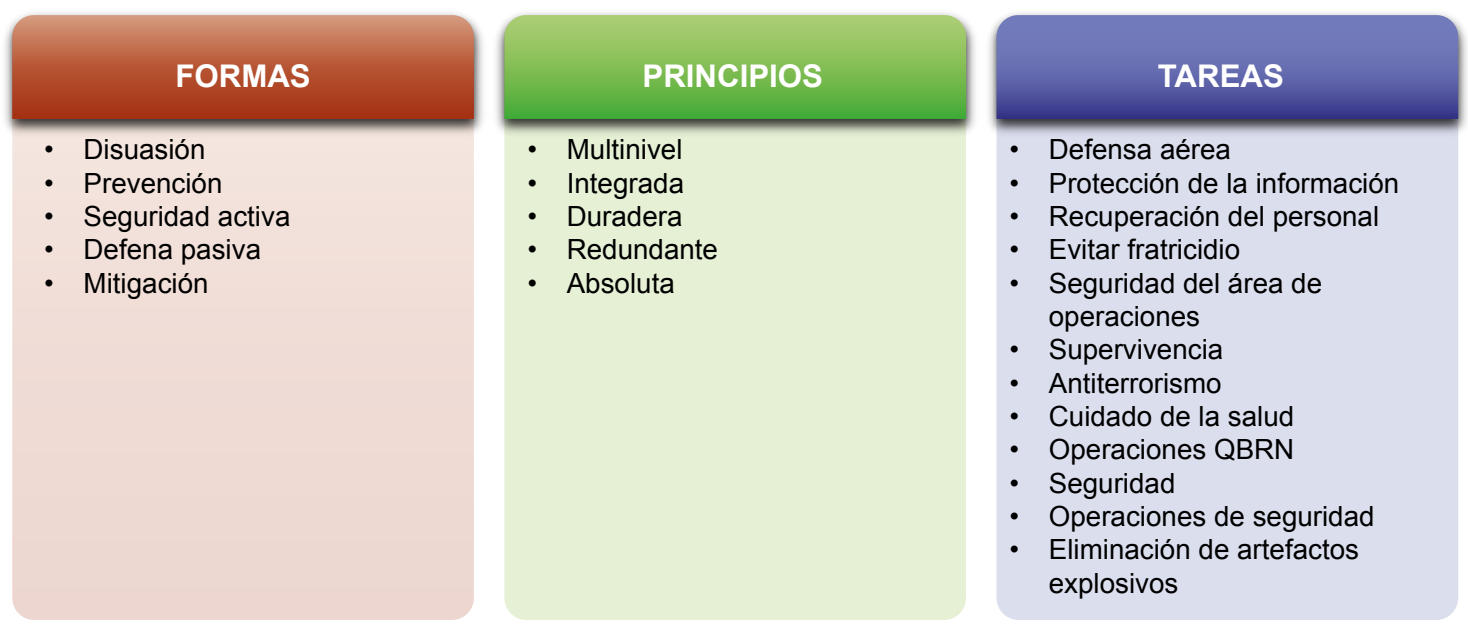

Figura 2. Conceptos rectores de la Protección. (Fuente: elaboración propia).

Los principios y formas de la protección proporcionan el contexto general y guía para el planeamiento y servirán de marco para la asignación de tareas y misiones para los elementos subordinados. Asimismo, las doce tareas señalan los aspectos claves a planificar y ejecutar para conformar un sistema de protección que permita asegurar integralmente las fuerzas, su misión y operaciones.

Las definiciones anteriores, muestran que el tradicional concepto de Seguridad por el cual se llevan a cabo dichas operaciones, está íntimamente ligado a la idea de Protección y sin dudas, ambos preceptos se integran y complementan, ya que no puede concebirse una operación de Protección sin tener en cuenta las normas de seguridad o la ejecución de operaciones en tal sentido. Del mismo modo, no puede hablarse de asegurar una fuerza, sus instalaciones, las operaciones e información sino se contempla la adopción de medidas protectoras que eviten o disminuyan los riesgos o efectos del accionar enemigo.

\section{La Protección durante la planificación, preparación y ejecución}

Las tareas de Protección se desarrollarán durante las tres fases de cualquier operación militar, iniciando con la planificación y siguiendo con la preparación y posterior ejecución de la misma. Por ello, la Protección no consistirá únicamente en planificar que medidas o acciones se llevaran a cabo, sino que éstas se irán actualizando permanentemente a medida que la operación apoyada se va desarrollando; es por esto que será un proceso de carácter cíclico y permanente. 
Esta actualización se materializa a través de la evaluación constante de la situación, el grado de desempeño y eficiencia de las medidas adoptadas y de los nuevos lineamientos que el comandante imponga.
El funcionamiento adecuado y eficiente del mencionado ciclo, implica considerar los siguientes aspectos y consideraciones:

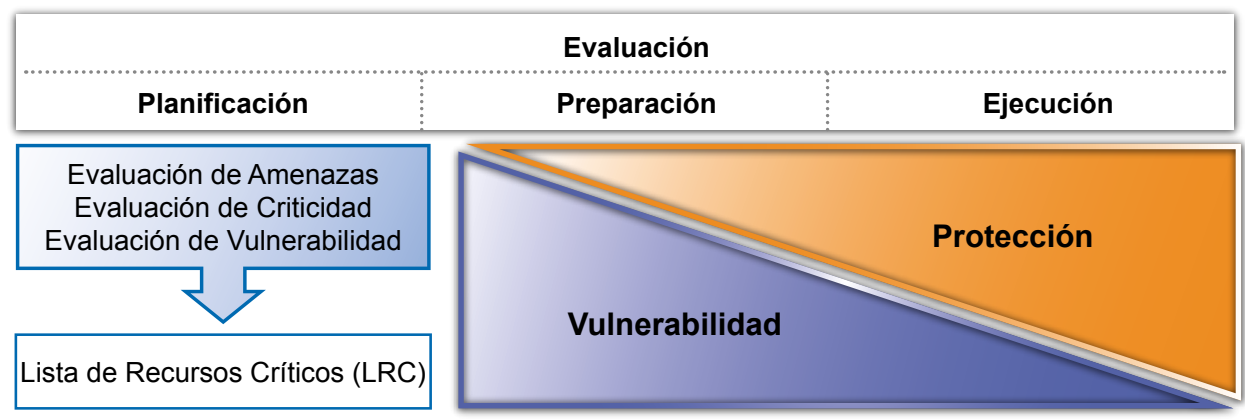

Figura 3. Proceso de evaluación de la Protección. (Fuente: reglamento Protection (FM 3-37), U.S. Army, 2009).

\section{Planificación}

Del mismo modo que se planifica cualquier operación militar, la metodología del Proceso Militar para la Toma de Decisiones (PMTD) será de plena aplicación y utilidad.

Es por eso que la planificación de las Operaciones de Protección, implica básicamente la identificación y evaluación de las probables amenazas, peligros y riesgos para luego establecer cuál es el grado de criticidad y vulnerabilidad de las fuerzas, instalaciones, información y medios críticos y prioritarios a proteger para cada fase de la operación militar que se vaya a desarrollar. Además, supone establecer cuáles son las medidas de prevención necesarias e incluirlas dentro de un esquema integral de protección.

Todo esto permite establecer cuatro factores claves a observar durante el planeamiento:
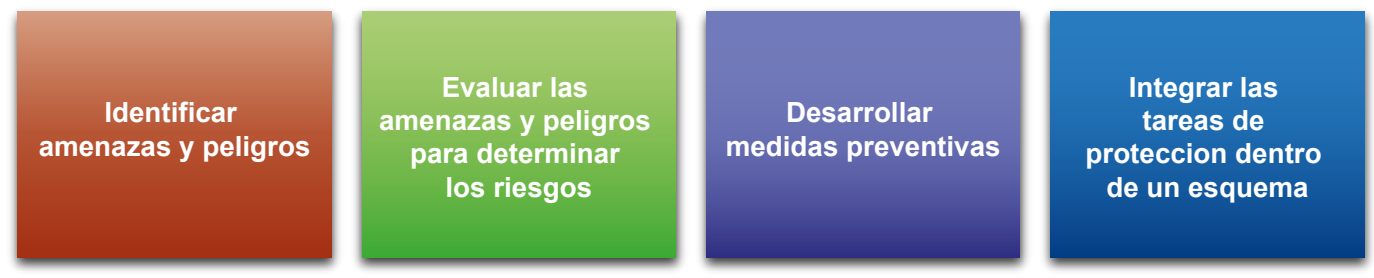

Figura 4. Aspectos claves para el planeamiento. (Fuente: elaboración propia).

Como todo proceso de planificación, será de carácter dinámico y permanente y deberá contemplar tanto la fase preparatoria como la ejecución de la operación en cuestión.
Sin embargo, la planificación de la Protección tendrá sus particularidades y características específicas para lo cual seguirá el consiguiente proceso de carácter cíclico:

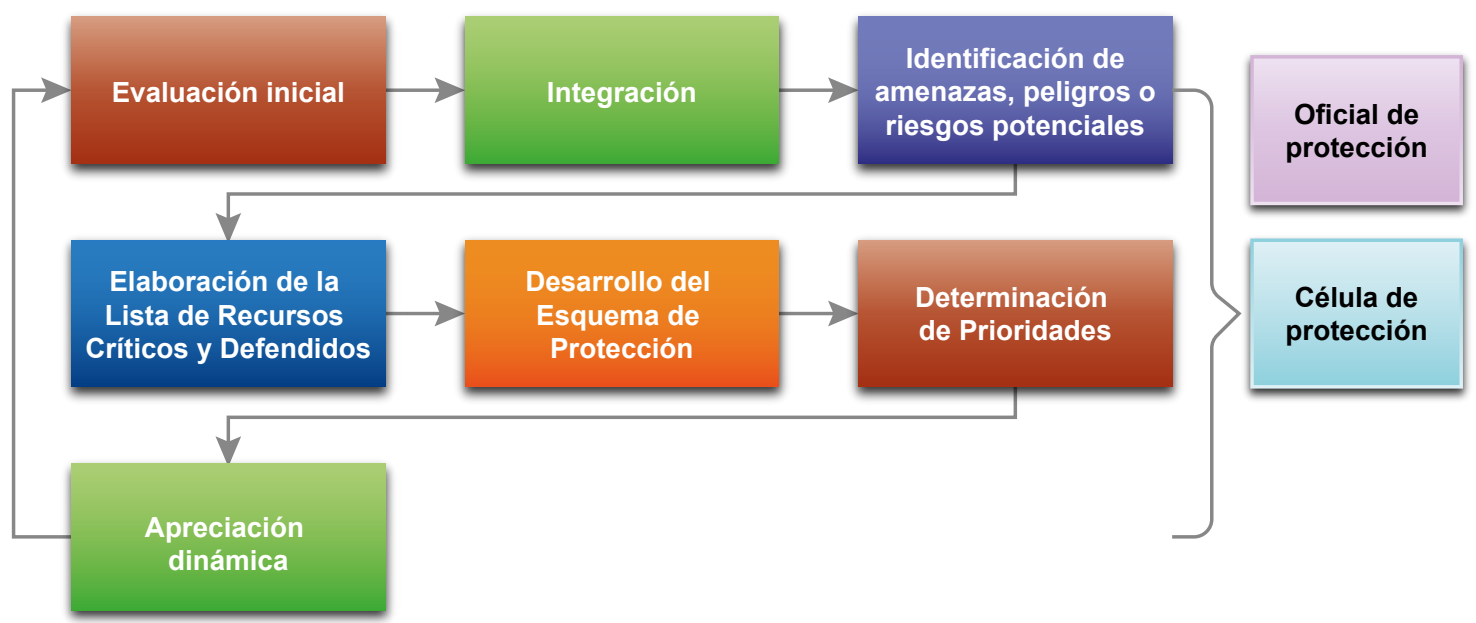

Figura 5. Secuencia de planeamiento de la Protección. (Fuente: elaboración propia). 
- Evaluación inicial:

En este primer paso se determinará el grado de criticidad de los recursos propios para cada fase de la operación táctica principal, estableciendo que recursos deben ser protegidos (Recursos Críticos) y cuáles de esos recursos se pueden proteger con los medios disponibles (Recursos Defendidos). Toda esta evaluación inicial se hará en base a los criterios y prioridades fijados por el Comandante.

\section{- Integración:}

Esta instancia contempla dos grandes tareas, primeramente realizar una adecuada selección de los objetivos probables del enemigo y luego el proceso de Gestión del Riesgo.

La selección de los objetivos probables del enemigo se obtendrá tras efectuar una detallada Preparación de Inteligencia del Campo de Batalla (PICB)

Luego, con el listado de los probables objetivos enemigos en la mano, se llevara a cabo el proceso de Gestión del Riesgo, mediante el cual se identifican, evalúan y controlan los riesgos derivados de las diferentes variables operacionales determinadas durante el PMTD. Esta gestión del riesgo permite determinar los recursos críticos, medios disponibles para la protección de ellos y que medidas de seguridad o de defensa son necesarias.

Adicionalmente, el proceso de evaluar los riesgos le proporciona al comandante un marco desde el cual poder establecer que riesgos está dispuesto a asumir y sobre cuales se adoptarán medidas protectoras para reducirlos o evitarlos.

- Identificación de amenazas, peligros o riesgos potenciales:

El reglamento colombiano de Protección (2017) define a las Amenazas como "cualquier combinación de actores, entidades o fuerzas que tienen la capacidad y la intención de afectar las fuerzas propias", a los Peligros como la "condición con el potencial de causar lesión, enfermedad o muerte del personal; daño o pérdida de equipo o propiedad; o la degradación de la misión" y a los Riesgos como la "probabilidad de pérdida o daño relacionado con uno o varios peligros".

En virtud a estos tres factores, en este paso se identifican y evalúan todas aquellas amenazas hostiles, no hostiles o derivadas de las condiciones del ambiente geográfico particular así como los potenciales peligros y riesgos que rodean a la misión, esta evaluación se realizará en función a las doce tareas propias de la Protección. También se debe evaluar la Criticidad, Vulnerabilidad y Capacidad de los propios recursos.

- Evaluación de Criticidad: permite establecer cual es el impacto en la propia fuerza de la afec- tación o destrucción por parte del enemigo de los recursos propios, así como su capacidad de recuperación.

- Evaluación de Vulnerabilidad: determina la magnitud del efecto de una amenaza, peligro o riesgo contra una instalación, personal o unidad y servirá para establecer qué medidas o medios serán necesarios para mitigar los efectos de la acción enemiga.

- Evaluación de Capacidades: se realiza sobre las organizaciones que conforman la fuerza y permite determinar su capacidad real para realizar tareas de protección, en base a los medios que dispone.

Un procedimiento sencillo y de fácil empleo para realizar estas tres evaluaciones, es el método CARVER de análisis de objetivos:

- Criticidad: grado de influencia que tiene la afectación del recurso para las propias fuerzas u operación.

- Accesibilidad: señala la facilidad del enemigo para afectar el recurso considerado.

- Recuperabilidad: capacidad o tiempo de recuperación del objetivo protegido.

- Vulnerabilidad: estará en función a la capacidad del enemigo de afectarlo y de los propios medios de protección.

- Efecto: señala la relación de efectos positivos o negativos que tendrá para las propias fuerzas y operaciones, su afectación por parte del enemigo.

- Reconocibilidad: indica el grado de facilidad que tendrá el enemigo para detectar el objetivo, en relación a las características del mismo y de las capacidades enemigas en tal sentido.

Una vez realizada la identificación y las tres evaluaciones, se elabora una lista completa de las amenazas, peligros y riesgos, junto con la probabilidad de ocurrencia de cada uno de ellos.

Este paso resulta de gran importancia puesto que de aquí surgirán elementos de juicio fundamentales para la elaboración y desarrollo de la siguiente instancia.

- Elaboración de la Lista de Recursos Críticos (LRC) y Recursos Defendidos (LRD):

Las LRC y LRD son elementos clave para la protección y se caracterizan por su dinamismo, por lo que deberán ser actualizadas permanentemente en función a la evolución de la situación táctica y de las nuevas prioridades que fije el comandante.

La LRC es una lista priorizada de los recursos propios, normalmente identificados para cada fase de la operación y aprobados por el Comandante y constituyen lo que "debe" ser protegido, mientras que la LRD deriva de la LRC y detalla que recursos "pueden" ser defendidos con los medios disponibles. 
La determinación de la LRC se realizará en base a los criterios de Valor (impacto de la pérdida sobre la propia fuerza y operación), Profundidad (proximidad en tiempo y distancia al enemigo), Impacto (grado de esfuerzo, costo y tiempo de reemplazo) y Capacidad (función y capacidad para operaciones actuales y futuras) (Ejército Nacional de Colombia, 2017, pág. 16).

- Desarrollo del esquema de protección:

Describe cómo las tareas de protección apoyan la intención y el concepto de la operación del Comandante y en base a su orientación, establece las prioridades de apoyo a las unidades para cada fase de la operación. Además, facilita la aplicación y enfoque de las tareas de protección.

- Determinación de Prioridades:

La criticidad, vulnerabilidad y capacidad de recuperación de los recursos a proteger, junto a la guía del Comandante, son los parámetros más importantes a la hora de establecer las prioridades de Protección, teniendo en cuenta que raramente se dispondrá de los medios necesarios para proteger adecuadamente a todos los recursos. Por ello, este paso será de fundamental importancia durante el proceso de planeamiento.

Asimismo, será una de las instancias más difíciles del proceso, puesto que por su carácter netamente subjetivo, estará sujeto a la experiencia, conocimientos y opiniones de quienes deban establecer las prioridades, las que luego serán puestas a consideración del comandante para su aprobación.

\section{- Apreciación dinámica:}

Esta actividad será de carácter permanente, no solo durante el planeamiento sino durante la preparación y ejecución de la operación táctica.

Proporciona una "imagen" actualizada de la situación, a partir de la información disponible, la misión y esquema de protección adoptado junto a la permanente actualización de las amenazas, peligros, riesgos, prioridades y medidas adoptadas, todo ello facilitará el control de la operación de
Protección y la adopción de nuevas disposiciones necesarias para asegurar su eficacia y alcanzar los resultados deseados.

Debido al carácter cíclico y permanente del proceso de planeamiento, tras haber finalizado la apreciación dinámica, nuevamente volverá a desarrollarse la Evaluación Inicial, en la medida que la situación evolucione.

Asimismo, durante todo el desarrollo del procedimiento de planificación, tendrá vital importancia el trabajo y desempeño del Oficial y de la Célula de Protección.

- Oficial de Protección:

Será el oficial, miembro del Estado Mayor Especial, específicamente capacitado para asesorar al comandante en todo lo referido a la protección, ejerciendo el mando de la célula de protección y coordinando el trabajo de los diferentes órganos de protección de los elementos subordinados, con el objetivo de lograr una adecuada sinergia entre los planes que cada uno de ellos lleva adelante y verificando que se encuentren alineados con las prioridades y planes vigentes.

- Célula de Protección:

Este órgano tendrá la responsabilidad de integrar, coordinar y sincronizar las tareas y actividades de protección, asesorando al comandante, así como coordinar la ejecución y el mantenimiento de las medidas de protección adoptadas durante todas las fases de la operación apoyada.

Es importante destacar que la célula de protección no planifica la operación, correspondiéndole esa tarea al Estado Mayor, mientras que la Célula de Protección proporciona información al PMTD mediante la integración de la evaluación de amenazas, peligros o riesgos con los elementos esenciales de información de las propias tropas, la LRC, y la LRD.

Este equipo de trabajo, estará conformado por especialistas en las diferentes doce tareas de protección y normalmente, se establecerá a nivel división y superiores.

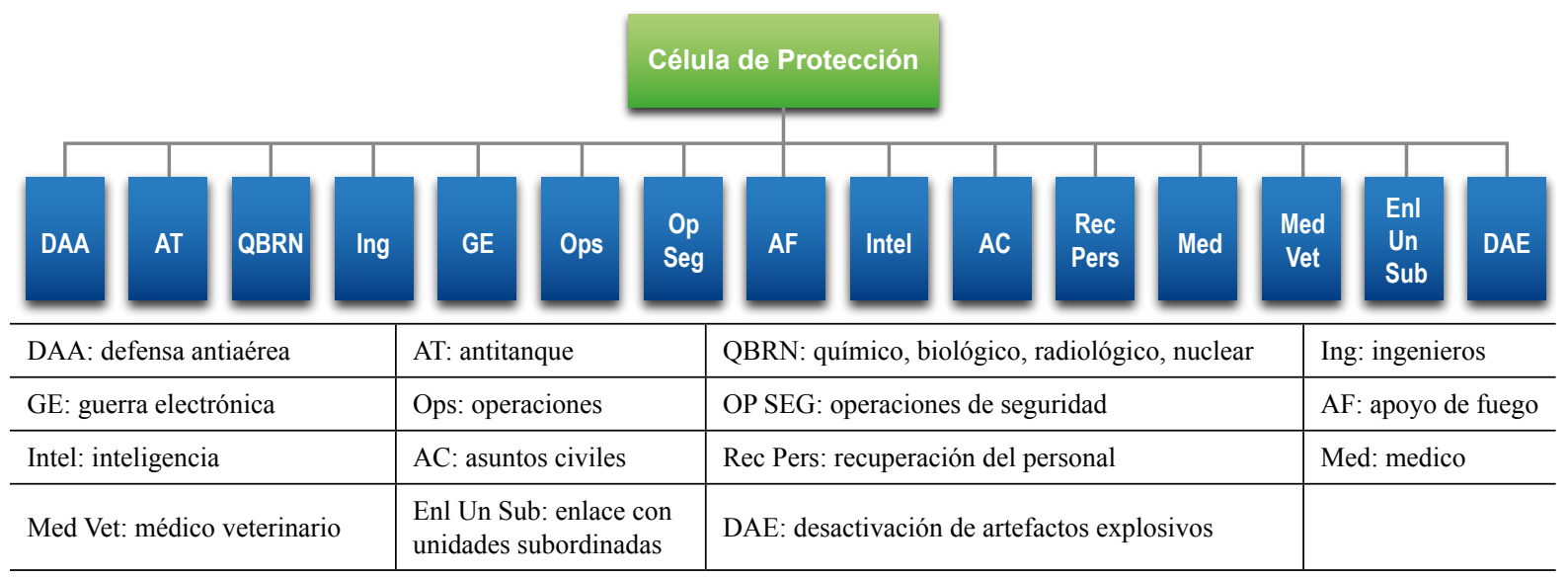

Figura 6. Composición de la Célula de Protección. (Fuente: elaboración propia). 


\section{La Protección durante la Preparación}

La preparación crea condiciones que mejoran las oportunidades para que la fuerza alcance el éxito; aunque durante este periodo normalmente se es más vulnerable a las acciones sorpresivas del enemigo. Por ello, la adopción de medidas de protección durante esta etapa será de vital importancia para que la fuerza llegue en las mejores condiciones a la fase siguiente, la ejecución.

En esta fase la protección se debe centrar en la disuasión del enemigo y en la prevención de todas aquellas acciones que este pueda realizar y que afecten directamente el poder de combate y la libertad de acción propia; o sea, la ejecución de las tareas de protección, en paralelo con las de preparación, ayudará a disminuir los riesgos. Mientras se preparan para las operaciones, los comandantes deben asegurar la integración y coordinación de las tareas y los sistemas de protección, a fin de salvaguardar puestos de mando, bases, rutas, equipos, información, infraestructura crítica, material bélico y personal. (Ejército Nacional de Colombia, 2017, pág. 58)

Adicionalmente, la adopción de medidas de defensa activa ayudará a negar la iniciativa al enemigo, mientras que la ejecución de medidas de defensa pasiva preparará a la fuerza contra los efectos negativos de las amenazas, peligros o riesgos, las que también facilitarán la atenuación de los posibles daños.

Durante la etapa de preparación, se llevarán a cabo fundamentalmente cuatro grandes actividades:

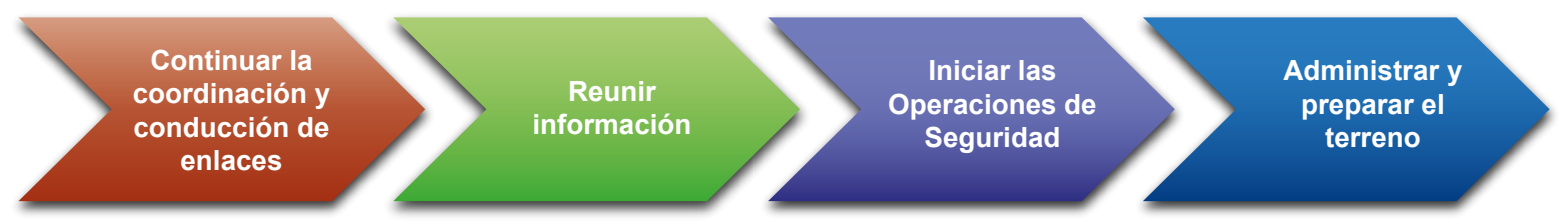

Figura 7. Secuencia de actividades de Protección durante la Preparación. (Fuente: elaboración propia).

- Continuar la coordinación y conducción de enlaces: Servirá para que los diferentes comandantes comprendan el rol y misión que tiene cada uno de sus elementos dentro del esquema de protección así como servir para evitar la duplicación de esfuerzos $\mathrm{y}$ asegurar un eficiente funcionamiento del sistema de protección.

Esta actividad será permanente y continua, manteniéndose durante la ejecución de la operación decisiva apoyada, la cual está "destinada a alcanzar el cumplimiento de la misión asignada, el punto focal sobre el cual el comandante efectúa el planeamiento de todas sus acciones asignando su especial atención y mayor prioridad" (Ejército Argentino, 2015, pág. 80) y por ende, será una de las principales tareas a desempeñar por la Célula de Protección.

- Reunir información:

El proceso de reunión de información es permanente y continuo en toda organización militar, es por ello que en las operaciones de Protección deberá orientarse el esfuerzo de obtención de información a llenar los vacíos informativos existentes en cuanto a las capacidades y objetivos del enemigo y de las propias vulnerabilidades, ya que ello facilitará la actualización de los planes y el mejoramiento del sistema de protección montado.

Como toda actividad de inteligencia, constituirá una tarea continua y permanente.
- Iniciar las Operaciones de Seguridad: Esta operación complementaria tiene como finalidad proporcionar la alerta temprana, otorgar tiempo y espacio para la reacción de la propia fuerza, preservar fuerzas, medios e instalaciones y negar información al enemigo (Ejército Argentino, 2015, pág. 192) y como tal, constituye una de las doce tareas de protección.

Por ello, estas operaciones deberán lanzarse cuando el grueso de las fuerzas inicia su preparación para la operación decisiva y mantenerse a lo largo de toda la siguiente fase de ejecución.

La detallada planificación y ejecución de estas operaciones resultará fundamental para el funcionamiento efectivo y eficiente del esquema de protección.

- Administrar y preparar el terreno:

Esta actividad implica la adopción de medidas de coordinación y control sobre el emplazamiento de las diferentes unidades subordinadas dentro del área de operaciones. Ello permitirá armonizar y sincronizar las diferentes tareas de protección, a la vez de economizar medios, evitar superposiciones, reducir el riesgo de fratricidio y en suma, lograr un funcionamiento más eficiente de todo el sistema de protección. 
Adicionalmente, la adopción de medidas de Control de Daño Zonal, que comprenden "las medidas preventivas y de control tomadas antes, durante y después de un ataque para reducir su efectos" (Ejército Argentino, 2015) y que por estas características, contribuirán a evitar o reducir los efectos de las posibles acciones del enemigo sobre las propias tropas, medios e instalaciones. Estas medidas, debidamente coordinadas e implementadas redundaran en un mejor funcionamiento y resultados de las medidas de protección adoptadas.

En esta etapa de Preparación, la Célula de Protección tendrá la tarea de asegurar que los controles y medidas de reducción de riesgo se implementen y se reflejen en los planes; asimismo deberán contemplarse los posibles cambios que se den en el tipo y grado de amenaza, lo que supone la actualización de los planes y medidas adoptados.

\section{La Protección en la Ejecución de las operaciones}

Las tareas y actividades de Protección serán aplicables a cualquier tipo de operación militar y por el carácter dinámico que ellas tienen, resultara esencial que las medidas de protección guarden el mismo ritmo y se actualicen al mismo tiempo que la operación principal lo hace.

Para ello, los comandantes de todo nivel deberán implementar medidas de coordinación y control que faciliten el funcionamiento del esquema de protección, así como asignar el personal, medios y recursos necesarios para que el plan de protección continúe vigente y en funcionamiento, ya que del mismo, dependerá en gran medida el éxito de la operación principal.

La adecuada coordinación y sincronización de las medidas protectoras y de mitigación de daños adoptadas serán la clave del éxito del plan de protección, ya que permitirán reducir o evitar los efectos del accionar enemigo. Esto facilitará que los comandantes economicen fuerzas y puedan mantener un adecuado grado de libertad de acción e iniciativa.

Es por ello, que esta etapa, caracterizada por rápidos cambios de situación, gran volumen de información y por la fricción propia del combate, impondrá que la Célula de Protección realice la evaluación de la situación en forma permanente y veloz, actualizando las amenazas, peligros y riesgos y determinando si las LRC y LRD necesitan modificarse, para así estar en condiciones de proponer al comandante las modificaciones necesarias para que el sistema de protección continúe cumpliendo su función de manera eficiente.

\section{Las operaciones de Protección en la doctrina militar sudamericana}

Como se mencionara anteriormente, en la primera década del siglo XXI el ejército de EE.UU incorporó en su doctrina las operaciones de Protección de la Fuerza, como forma de materializar los conceptos ya existentes y que hacían referencia a la protección como Función de Combate.

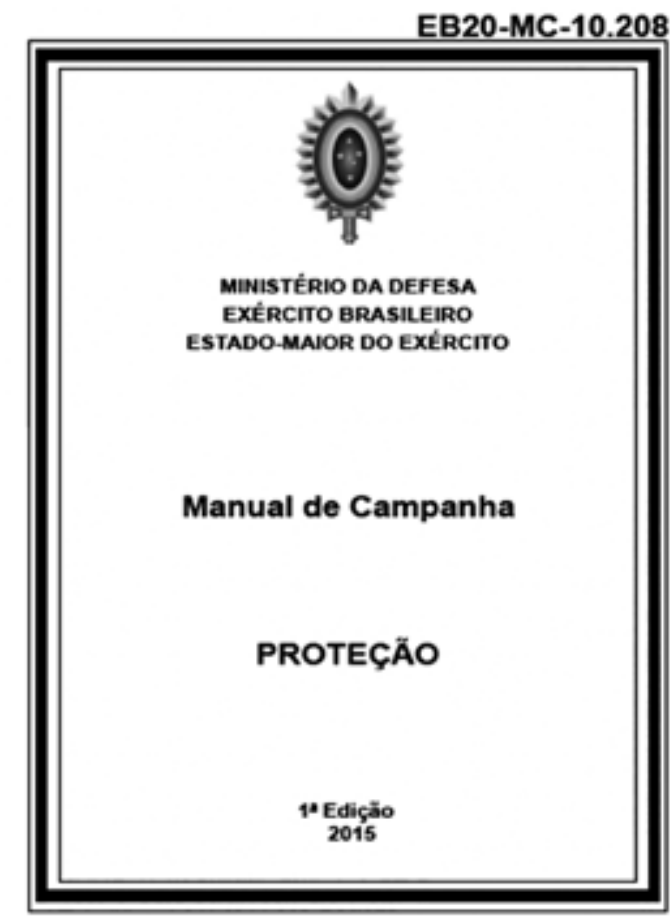

Figura 8. Reglamento de Protección brasileño. (Fuente: Ejército Brasileño).

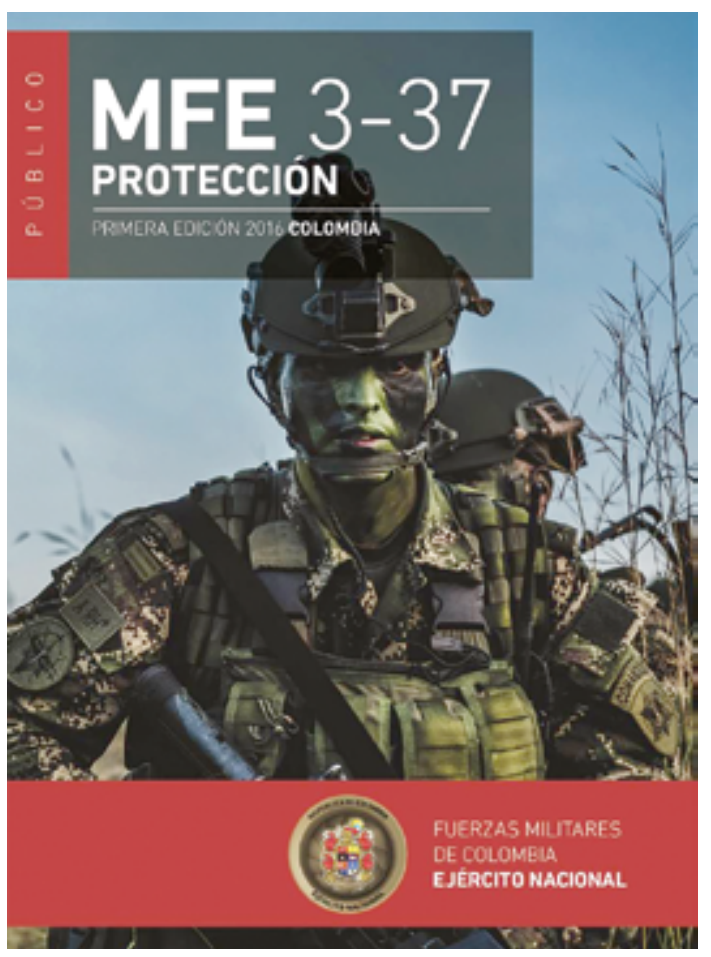

Figura 9. Reglamento de Protección colombiano. (Fuente: Ejército Colombiano). 
De ese modo, en 2009 sale a la luz el reglamento de Protección (FM 3-37) lo cual es repetido por la OTAN en su doctrina operativa en el año 2015.

Dentro de nuestro subcontinente, el concepto de Protección ha sido incorporado en la doctrina militar de varios países, entre ellos Argentina, Brasil y Colombia, como una de las denominadas "Funciones de Combate" (Argentina y Brasil) o como "Función de Conducción de la Guerra" (Colombia).

Sin embargo, tan solo Brasil y Colombia han incorporado las nuevas teorías referidas a las operaciones de Protección como una más de las operaciones militares de configuración, las que tienen el "propósito de crear o preservar las condiciones favorables para el éxito de la operación decisiva" (Ejército Argentino, 2015, pág. 81 , que las fuerzas pueden llevar a cabo en apoyo a la operación principal.

En 2015 el ejército brasileño pone en vigencia su reglamento de Protección (EB20-MC-10.208), mientras que Colombia lo hace en el año 2017. De esta manera, ambos países han desarrollado su propia doctrina con la finalidad de servir de guías para los comandantes y sus Estados Mayores en la planificación y conducción de esas operaciones particulares.

Sin embargo, si bien ambos países han tomado la doctrina americana y de la OTAN como base para la suya propia, se observan ciertas diferencias sustanciales a la hora de definir las tareas o actividades de protección y fundamentalmente, en el proceso de planeamiento.

Por un lado, la doctrina colombiana adopta y refleja fielmente los conceptos y teorías establecidos por la doctrina americana y de la OTAN, incorporando íntegramente las definiciones, tareas, formas, principios, etapas, procesos de evaluación y planeamiento, activi- dades en la preparación y ejecución y en los órganos que llevaran a cabo todas estas labores.

No obstante, el reglamento colombiano postula una serie de matrices y tablas guías para las diferentes etapas de la operación de protección, los que constituyen elementos que materializan claramente cuáles son las tareas a realizar, su secuencia, coordinación y los productos -documentos, consideraciones, disposiciones, etc.- que deben ser elaborados al final de cada etapa y que conforman el fruto del trabajo del estado mayor y de la célula de protección.

Estas matrices y tablas, las que es necesario destacar, no existen en los manuales estadounidenses y de la OTAN, se constituyen en elementos fundamentales para comprender acabadamente como deben pensarse y ejecutarse las operaciones de protección. Además, por su formato sencillo, facilitan el trabajo del estado mayor y de la célula de protección, al constituirse en guías prácticas para el desarrollo de todas las actividades de planeamiento y supervisión de las acciones.

Por estos motivos, el reglamento colombiano sobresale por la profundidad, claridad y desarrollo de los diferentes conceptos así como por el grado de detalle con que se explicitan todas y cada una de las etapas y tareas propias de la función Protección.

Por otra parte, el manual "Proteção"del ejército brasileño, adopta conceptos básicos como la definicion de protección, los principios, el oficial y la célula de protección y algunas de las tareas propias de esta funcion. Sin embargo no toma el procedimiento de planeamiento americano y como documentos de guia para el estado mayor, solo proporciona un modelo de anexo de protección a la orden o plan de operaciones y una matriz de sincronización de las tareas de protección. Igualmente, se destaca por lo breve y conciso de sus conceptos.

\begin{tabular}{|c|c|c|}
\hline & Doctrina EEUU-OTAN-Colombia & Doctrina de Brasil \\
\hline Tareas & $\begin{array}{l}\text { Defensa aérea } \\
\text { Protección de la información } \\
\text { Recuperación del personal } \\
\text { Evitar fratricidio } \\
\text { Seguridad del área de operaciones } \\
\text { Supervivencia } \\
\text { Antiterrorismo } \\
\text { Cuidado de la salud } \\
\text { Operaciones QBRN } \\
\text { Seguridad } \\
\text { Operaciones de seguridad } \\
\text { Eliminación de artefactos explosivos }\end{array}$ & $\begin{array}{l}\text { Contra inteligencia } \\
\text { Defensa antiaérea } \\
\text { Defensa QBRN } \\
\text { Anti terrorismo } \\
\text { Guerra electrónica } \\
\text { Guerra cibernética } \\
\text { Búsqueda y salvamento } \\
\text { Seguridad del área de operaciones }\end{array}$ \\
\hline
\end{tabular}

Tabla 1. Comparación de tareas entre la doctrina EEUU-OTAN-Colombia y Brasil. (Fuente: elaboración propia). 
Del análisis de la tabla anterior se puede determinar que existen tareas que la doctrina brasileña no ha contemplado como inherentes a la función Protección, probablemente esto se deba a que ellas son actividades y responsabilidades propias de otras organizaciones, funciones o sistemas que operan en el campo de combate, a saber: las tareas de recuperación del personal y cuidado de la salud son propias del sistema de sanidad y es responsabilidad de este sistema su implementación y ejecución, así como las tareas de apoyo a la supervivencia y la eliminación de artefactos explosivos son responsabilidad de los elementos del arma de Ingenieros y del sistema que ésta conforma en apoyo a las operaciones.

Del mismo modo, el reglamento brasileño contempla su propio procedimiento de planeamiento, el que difiere totalmente del método colombiano pero que guarda una gran similitud con la secuencia metodológica del PMTD:

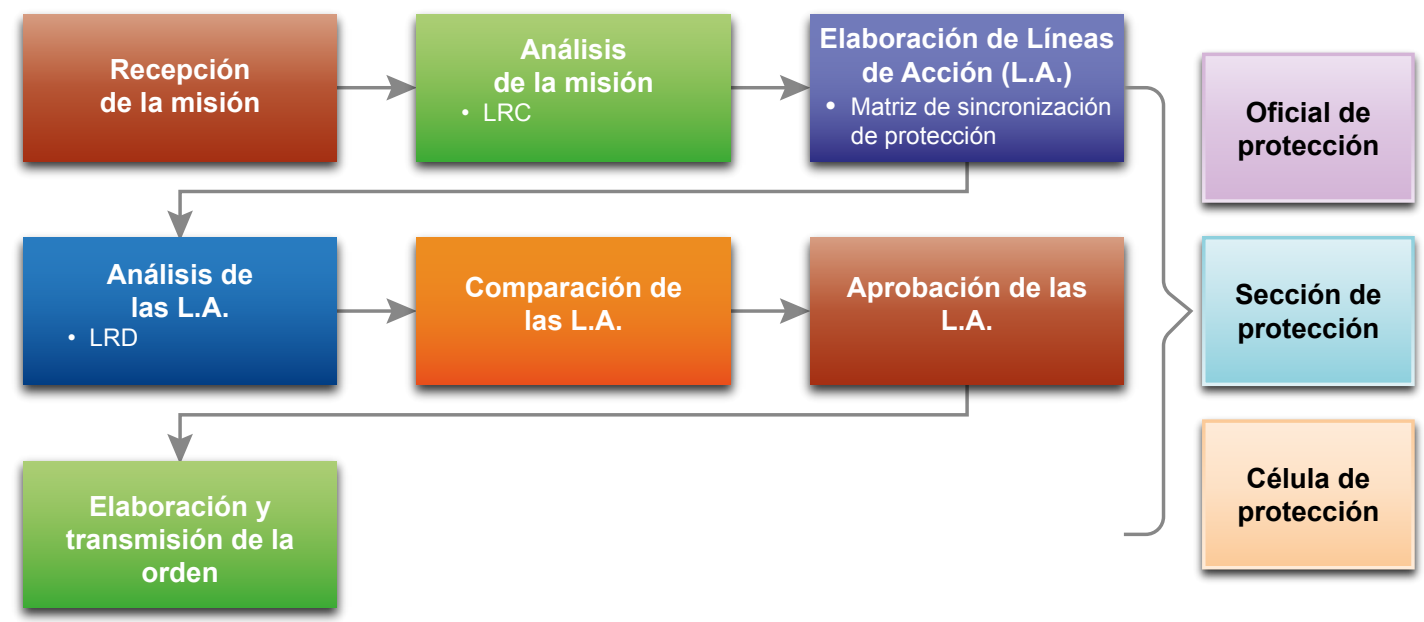

Figura 10. Secuencia de planeamiento brasileño. (Fuente: elaboración propia).

Además de estas diferencias metodológicas con el procedimiento colombiano, la doctrina brasileña incorpora la figura de la Sección de Protección, la que tendrá la responsabilidad de planear y coordinar todo lo referido a las operaciones de protección (Exército Brasileiro, 2015, pág. 21); dejando para la célula de protección la función de coordinar las actividades de protección de los sistemas y medios subordinados destinados a preservar a la fuerza.

Esto constituye una de las diferencias más significativa entre ambas doctrinas, puesto que en el caso colombiano, la planificación de las operaciones de protección es una tarea adicional a llevar a cabo por el estado mayor, disponiendo para ello del asesoramiento y asistencia de la célula de protección. Mientras que en el caso brasileño, la planificación será responsabilidad y tarea de la sección de protección -bajo el mando del oficial de protección- lo que facilitará el trabajo del estado mayor al quitarle el peso significativo que implica planear este tipo de operaciones.

En lo que respecta a la célula de protección, el modelo brasileño le confiere similares responsabilidades y funciones que las prescriptas por el manual colombiano.

Sin embargo, mas allá de las diferencias conceptuales y metodológicas, es menester destacar que la puesta en vigencia de los reglamentos considerados significa un importante avance en materia de pensamiento mi- litar por parte de ambos países, quienes con esta actualización doctrinaria en paralelo con lo existente en los países más desarrollados, muestran la creciente importancia que tanto Brasil como Colombia otorgan a la protección y seguridad de sus ejércitos y que va más allá del concepto previo de seguridad y de las operaciones derivadas de ella.

\section{Conclusiones}

Las operaciones de Protección constituyen una importante y novedosa actualización doctrinaria, fruto de la permanente evolución del arte de la guerra y de las capacidades que las fuerzas militares convencionales y no convencionales van incorporando.

A raíz de esto, se ha hecho necesario pensar no tan solo en asegurar las tropas e instalaciones contra las acciones del enemigo sino también proteger valores intangibles como la información y la moral del combatiente.

Asimismo, las operaciones de protección no se limitan en su aplicación tan solo al nivel táctico de la conducción militar sino que también pueden y deben ser puestas en práctica en los otros dos niveles superiores: el operacional y el estratégico militar, pues en todas las instancias habrá recursos y medios que proteger.

Esto último, junto a la creciente evolución tecnológica, también implica que las operaciones de protección no solo se limitarán al campo de batalla tradicional 
y tridimensional de la llamada "Batalla Aeroterrestre" vigente hasta finales del siglo $\mathrm{XX}$ sino que deberán considerarse y ejecutarse también, en el espacio exterior, el ciberespacio, el entorno electromagnético, la dimensión cognitiva de la guerra y el ámbito de la información (South, 2017). Todo ello no es otra cosa que el nuevo concepto de las denominadas "Operaciones Multi Dominio".

Todas estas consideraciones suponen una creciente dificultad para llevar todas estas tareas y funciones adelante, por lo que resultará necesario educar hombres y organizaciones en la planificación y conducción de las tareas y actividades de protección, incorporando a los Estados Mayores la figura del Oficial de Protección y de la Célula de Protección, quienes asesoraran al Comandante, colaboraran en la redacción de los planes, propondrán medidas de acción, integraran los planes y coordinaran las medidas de protección adoptadas.

Esto significa que deberemos repensar nuestras organizaciones y estructuras de mando para incorporar la protección como una de las operaciones de configuración más importantes y con ello, incrementar el grado y capacidad de supervivencia de las fuerzas, preservar medios e información y de ese modo coronar con éxito la operación decisiva.
De la misma manera, resulta imperativo que las fuerzas armadas de los países que aún no lo han hecho, incorporen esta nueva teoría militar a sus cuerpos doctrinarios, porque como se ha expuesto, el continuar operando únicamente bajo el tradicional pero insuficiente concepto de Seguridad dejará a nuestras fuerzas con un alto y creciente grado de vulnerabilidad y expuestas a las diferentes acciones de un enemigo cada vez mejor dotado de un amplio espectro de capacidades tecnológicas y armamentísticas.

Como se ha mostrado, las fuerzas armadas de los países desarrollados se encuentran trabajando en esta problemática desde hace ya más de una década y los ejércitos de Brasil y Colombia han sido los precursores de la materia a nivel regional, señalando la senda que el resto, más temprano que tarde, habrá de seguir.

"Debemos mantener nuestra mente alerta y receptiva a la aplicación de métodos y armas que no hayamos vislumbrado. La próxima guerra será ganada en el futuro, no en el pasado. Debemos mantenernos a la vanguardia o permitir que seamos relegados". General Douglas Mac Arthur.
[1] Berkebile, R. E. (2016). Thoughts on force protection. Joint Doctrine Quarterly (81), 140-148.

[2] Ejército Argentino. (2015). Conducción de las Fuerzas Terrestres, ROB 00-01. Buenos Aires: Departamento Doctrina.

[3] Ejército Ecuatoriano. (2015). Manual de Conducción Militar, MI3-TASE1-02. Quito: Comando de Educación y Doctrina del Ejército.

\section{REFERENCIAS}

[4] Ejército Nacional de Colombia. (2017). Protección, MFRE 3-37. Bogotá: Comando de Educación y Doctrina.

[5] Exército Brasileiro. (2015). Proteção, EB20-MC-10.208. Brasilia: Centro de Doutrina do Exército.

[6] Joint Chief of Staff. (2017). Joint Operations, JP 3-0. Washington: The Joint Staff.
[7] NATO. (2015). Allied Joint Doctrine for Force Protection, AJP 3-14. Londres: Nato Standarization Office.

[8] South, T. (31 de diciembre de 2017). Army Times. Recuperado el 12 de febrero de 2020, de U.S. Army Training and Doctrine Command: https://www.armytimes.com/news/ your-army/2017/12/31/new-in2018-army-pushes-forward-onmultidomain-battle-new-way-to-fight/

[9] U.S. Army. (2009). Protection, FM 3-37. Washington: Headquarters Department of the Army. 